\title{
Photometric periods of HN Peg from multichannel analysis ${ }^{\star}$
}

\author{
J. You ${ }^{1}$ and R. Duemmler ${ }^{2}$ \\ 1 Astronomy Division, Department of Physical Sciences, PO Box 3000, 90014 University of Oulu, Finland \\ 2 Frankenallee 201, 60326 Frankfurt/Main, Germany
}

Received 9 October 2000 / Accepted 22 November 2004

\begin{abstract}
We applied the method of three-stage weighted multichannel period analysis (MPA) to photometric observations of HN Peg obtained in 1992-99. We detected two periods: the longer one stayed almost constant near 5.1 but appeared only during the observing seasons in 1993-98, while the shorter one was present for all observing seasons, having fluctuations within $4.4-4$ d. 8 .

If the two periods are interpreted as caused by differential rotation, we may have detected two distinct latitude zones of spot activity, one where spots were confined to within a very narrow range of latitude, and another where the latitude range may be somewhat broader. The existence of two latitude zones of activity in this young solar analogue closely matches what is seen in the Sun. However, the presence of two periods may also be caused by long-lived active areas moving in longitude.

We use HN Peg as a test case for our new MPA statistical method. Because of the limited quality of the data and the complexity of stellar activity our results are tentative, leaving space also for other interpretations.
\end{abstract}

Key words. methods: data analysis - stars: individual: HN Peg - stars: rotation - stars: activity - stars: starspots

\section{Introduction}

Information from photometric and spectroscopic variability due to starspot activity and its rotational modulation is of great importance in understanding the chromospheric activity and the magnetic field cycle of sun-like stars. As a nearby young solar analogue (see Gaidos 1998), HN Peg (HD 206860, HR 8314; average $V=6.0, B-V=0.5 ; \mathrm{m} 0 \mathrm{~V}$ ) is a useful candidate for testing new analysis methods. It is also one of the stars identified by Wilson (1978) as exhibiting variations in the Ca II H\&K emission flux.

Vaughan et al. (1981) used the modulation observed in the Ca II $\mathrm{H} \& \mathrm{~K}$ emission flux to deduce a rotation period of 4.7 for HN Peg. This period is consistent with the spectral type and with the spectroscopically determined value of $v \sin i=$ $11 \mathrm{~km} \mathrm{~s}^{-1}$ obtained by Kraft (1967).

Baliunas et al. (1985) analyzed the fluxes in the Ca II H\&K emission lines obtained in 1980, 1981 and 1982. They detected periods of $4.65 \pm 0.01$ and $5.00 \pm 0.02$ in 1980 , $4.662 \pm 0.007$ and $4.91 \pm 0.01$ in 1981 , and $4.675 \pm 0.005$ in 1982. They explained the two distinct periods as arising from active regions at different latitudes rotating differentially with respect to each other. An alternative explanation is the growth and decay of active regions with subsequent birth of new active regions at stellar longitudes different from the original sites of activity. While the rotational period remains the same in this case, the change in longitude introduces a phase shift: this shift

* Based on observations collected at the Mt. Maidanak (Uzbekistan) and Fairborn (USA) observatories. can lead to period-finding algorithms identifying more than one period, among which the true rotation period may not even appear. Baliunas et al. (1985) concluded that in HN Peg the fractional differential surface rotation would be at least $5 \%$, and that the nearly constant shorter period through all three years implies that an active zone persisted for the three years and modulated the chromospheric emission.

Donahue et al. (1996) analyzed the fluxes in the Ca II H\&K emission lines obtained over 12 years since 1980. Their results suggest the presence of surface differential rotation in the period range $4.57-5.30$, with the exception of 5.7 in 1984.

Gaidos et al. (2000) give their preliminary analysis of the observations of HN Peg from 1993 to 1998 in Strömgren $b$ and $y$ acquired with a $75 \mathrm{~cm}$ automatic photoelectric telescope (APT; The same data, extended until the end of 1999, constitute the second data set used in this paper). Their results show that the most significant period of HN Peg varies from year to year: $4.73 \pm 0.01$ in $1993,4.64 \pm 0.01$ in $1994,5^{\mathrm{d}} .10 \pm 0.01$ in $1995,5^{\mathrm{d}} .18 \pm 0.01$ in 1997 , and $4.68 \pm 0.04$ in 1998.

From their photometric and spectroscopic observations of HN Peg, Frasca et al. (2000) found a similar period of 4.74 . They used it to construct a 3D structure of the external atmosphere of HN Peg. The structure shows two spotted regions at different latitudes.

From the photometric observations of HN Peg in Gaidos et al. (2000), in Frasca et al. (2000) and their Villanova photometry (Messina \& Guinan 2002), Messina \& Guinan (2003) found that the HN Peg photometric period varied in a range 
Table 1. Observations of HN Peg together with periods and amplitudes derived from the Mt. Maidanak data. The entries are: the beginning and ending time of the season (S) as Heliocentric Julian date ( $\left.\mathrm{HJD}_{\text {start }}-\mathrm{HJD}_{\text {end }} ; 2440000 .+\right)$, the calendar months $\left(\mathrm{CD}_{\text {start }}-\mathrm{CD}_{\text {end }}\right)$, the time span $T_{\text {span }}$ in days, the number of observations $\left(N^{U}, N^{B}, N^{V}, N^{R}\right)$ in each channel, the final, refined periods $P$ with their error estimates $\sigma$ from the multichannel $(U B V R)$ nonlinear modeling (NLM), and the amplitudes of the single channels $U, B, V, R$ from the fit of a multiple period model.

\begin{tabular}{ccccccccccccc}
\hline \hline $\mathrm{S}$ & HJD & $\mathrm{CD}$ & $T_{\text {span }}$ & $N^{U}$ & $N^{B}$ & $N^{V}$ & $N^{R}$ & $P \pm \sigma$ & Amp. & Amp. $^{B}$ & Amp. $^{V}$ & Amp. $^{R}$ \\
\hline 1 & $8819-8969$ & $7-12,1992$ & 150 & 59 & 60 & 59 & 60 & $4.6261 \pm 0.0070$ & 0.0183 & 0.0188 & 0.0125 & 0.0123 \\
& & & & & & & & & & & & \\
2 & $9141-9287$ & $6-10,1993$ & 146 & 85 & 86 & 87 & 86 & $4.7483 \pm 0.0108$ & 0.0129 & 0.0098 & 0.0052 & 0.0063 \\
& & & & & & & & $5.1339 \pm 0.0087$ & 0.0089 & 0.0092 & 0.0115 & 0.0077 \\
3 & $9521-9625$ & $6-9,1994$ & 104 & 56 & 55 & 56 & 56 & $4.5734 \pm 0.0095$ & 0.0294 & 0.0285 & 0.0211 & 0.0128 \\
& & & & & & & & $5.1330 \pm 0.0224$ & 0.0175 & 0.0175 & 0.0145 & 0.0116 \\
4 & $9934-10044$ & $8-11,1995$ & 110 & 57 & 58 & 58 & 58 & $4.7062 \pm 0.0177$ & 0.0176 & 0.0152 & 0.0128 & 0.0082 \\
& & & & & & & & $5.1578 \pm 0.0094$ & 0.0299 & 0.0259 & 0.0222 & 0.0190 \\
\hline
\end{tabular}

of 4.59 to 5 d 17 from 1993 to 1998 . They interpreted the period variation as following from the modulation of the HN Peg surface differential rotation, this modulation being due to the existence of active latitude belts migrating on the star during its activity cycle.

The presence of multiple periods in late-type stars has been interpreted as the result of stellar (differential) rotation combined with active regions at different latitudes. These active regions are detectable via rotational modulation of their Ca II H\&K emission (e.g. Donahue et al. 1996) and the photometric brightness variations due to the presence of large, cool spots (e.g. Henry et al. 1995; Jetsu et al. 2000).

In this paper, we study an eight year photometric time series of HN Peg to derive properties of its rotation and chromospheric activity.

\section{Observations}

Two sets of photometric data have been used. The first set was obtained at the Mount Maidanak Observatory with the $60 \mathrm{~cm}$ telescope (data provided by V. S. Shevchenko). This $U B V R$ photometry was carried out from 1992 July through 1995 November, covering a total time interval of 1225 days. In Table 1 we give the time span and the number of observations in the $U, B, V$, and $R$ channels for each season. The observing procedures and reduction methods have been explained in detail by Shevchenko (1980), who gives error estimates for a single observation as 0.030 in $U$ and 0.015 for all the other filters.

The second data set was acquired with the T4 $75 \mathrm{~cm}$ automatic photoelectric telescope (APT) located at Fairborn Observatory in the Patagonia Mountains of southern Arizona. These are the same observations analyzed by Gaidos et al. (2000) with an additional season of observations provided by G. W. Henry (private communication). The Strömgren $b$ and $y$ observations extend from 1993 May until 1999 December and cover a time interval of 2417 days. The comparison star used was HD 209166 (=HR $8392=20$ Peg = ADS 15543 A). The observing and data reduction techniques are described in Henry (1999). Typical errors of a single observation were 0.0015 for both $b$ and $y$. Table 2 gives the time span and number of observations in $b$ and $y$ for each observing season. Figure 1 shows the time spans of the two data sets.

\section{Methods and results}

To search for periods in HN Peg we analyzed the Mt. Maidanak and APT data separately (both the entire data sets taken together and each individual season) with the method of multichannel weighted period analysis (MPA, You et al. 2000). This method finds common periods in all channels simultaneously. The three consecutive stages in the full algorithm are phase dispersion minimization (PDM), linear modeling (LM) and nonlinear modeling (NLM).

The results from MPA are only as valid as the assumptions about the light curves and underlying physical processes. In MPA, the periods are assumed to be constant throughout the data set being analyzed with no significant changes in the light curve shapes. Because of the nature of stellar activity, this assumption is surely false for data sets spanning several years. Therefore, we present the results from our MPA analysis separately for each season of the Mt. Maidanak and the APT data. However, we also discuss the results from the application of MPA to the two complete data sets in Sect. 4.

In the application of MPA to HN Peg, the first stage of PDM is used to find the significant time scales in the $D(f)$ spectrum, where $f=1 / P$ is the frequency and $D$ is the weighted phase dispersion, i.e. the sum of weighted squared differences between measurements subsequent in phase computed for the trial period $P$. The first significant period, defined by the deepest minimum in the multichannel $D(f)$ spectrum, is then refined through the LM and NLM stages (see below; and You et al. 2000). The identification of a second significant period is made by analyzing the residuals, which are obtained in the NLM stage by subtracting the first period fitted to the original data with a two-harmonic model. Here, sinusoid functions in the fitting model are chosen up to the second order, because of the probable complexity of the light curves and the limitation of the number of observations. If a second significant period is found, it is refined again using LM and NLM fits to the original 


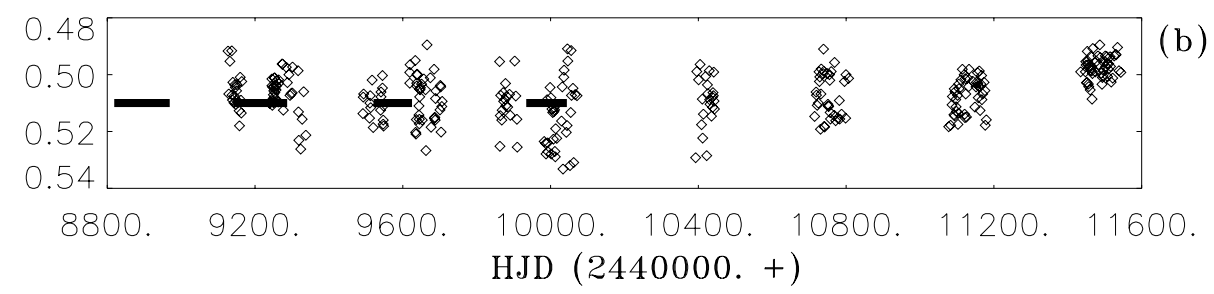

Fig. 1. Mt. Maidanak and APT observations. The diamonds are APT observations in $b$ (differential magnitudes with respect to the comparison star); the thick horizontal bars represent the time intervals of the Mt. Maidanak observations. The Mt. Maidanak data sets S2, S3 and S4 overlap with the APT data sets s2a, s2b, s3a, s3b, s4a and s4b. See details in Tables 1 and 2.

Table 2. Observations of HN Peg together with periods and amplitudes derived from the APT data. The table entries are as in Table 1. Here, for each season the number of observations $\left(N_{\mathrm{obs}}\right)$ for both $b$ and $y$ is identical. The season index numbers $(\mathrm{S})$ start from 2 , in order to correspond to the Mt. Maidanak data sets.

\begin{tabular}{|c|c|c|c|c|c|c|c|}
\hline $\mathrm{S}$ & HJD & $\overline{C D}$ & $\overline{T_{\text {span }}}$ & $N_{\text {obs }}$ & $P \pm \sigma$ & Amp. $^{b}$ & Amp. ${ }^{y}$ \\
\hline $2 a$ & $9127-9164$ & $5-6,1993$ & 37 & 22 & $4.6346 \pm 0.0417$ & 0.0142 & 0.0135 \\
\hline \multirow[t]{3}{*}{$2 b$} & $9240-9338$ & $9-12,1993$ & 98 & 43 & $4.6288 \pm 0.0107$ & 0.0191 & 0.0149 \\
\hline & & & & & $4.7548 \pm 0.0220$ & 0.0118 & 0.0091 \\
\hline & & & & & $5.0887 \pm 0.0197$ & 0.0086 & 0.0082 \\
\hline \multirow[t]{2}{*}{$3 a$} & 9492-9548 & $5-7,1994$ & 56 & 18 & $4.7156 \pm 0.0213$ & 0.0131 & 0.0114 \\
\hline & & & & & $\mathrm{a}$ & & \\
\hline \multirow[t]{2}{*}{$3 b$} & 9619-9707 & 9-12, 1994 & 88 & 39 & $4.6076 \pm 0.0099$ & 0.0234 & 0.0178 \\
\hline & & & & & $5.0999 \pm 0.0204$ & 0.0106 & 0.0105 \\
\hline $4 a$ & 9862-9910 & $5-7,1995$ & 48 & 19 & $\mathrm{~b}$ & & \\
\hline \multirow[t]{2}{*}{$4 \mathrm{~b}$} & $9983-10072$ & $9-12,1995$ & 89 & 36 & $4.6722 \pm 0.0244$ & 0.0156 & 0.0118 \\
\hline & & & & & $5.0994 \pm 0.0135$ & 0.0246 & 0.0228 \\
\hline \multirow[t]{2}{*}{5} & $10393-10444$ & $11-12,1996$ & 51 & 23 & $4.6108 \pm 0.0515$ & 0.0173 & 0.0145 \\
\hline & & & & & $5.0881 \pm 0.0584$ & 0.0195 & 0.0169 \\
\hline \multirow[t]{2}{*}{6} & $10715-10807$ & $9-12,1997$ & 92 & 36 & $4.4027 \pm 0.0183$ & 0.0048 & 0.0070 \\
\hline & & & & & $5.1642 \pm 0.0134$ & 0.0183 & 0.0172 \\
\hline 7 & $11078-11180$ & 9, 1998-1, 1999 & 102 & 46 & $4.6242 \pm 0.0098$ & 0.0150 & 0.0138 \\
\hline 8 & $11435-11544$ & $9-12,1999$ & 109 & 50 & $4.7908 \pm 0.0095$ & 0.0115 & 0.0111 \\
\hline
\end{tabular}

a: Insufficient data to determine a second period.

b: Insufficient data to determine any periods.

data. Both periods are then fixed in a LM fit of a model consisting of two periods (two harmonics for each) to the original data in order to determine the full set of amplitudes. Here, we use only the periods from the combined multichannel analysis, based on the requirement that the real periods are not wavelength dependent and the fact that the multichannel periods are more accurate than any of the single channel periods (You et al. 2000). Then, the two-period model is subtracted from the original data and the residuals are analyzed again. This procedure is repeated until no significant minima are left in the PDM spectrum.

PDM has been applied over the period search range $P_{\text {min }}=4.0$ to $P_{\max }=9.0$, although a preliminary analysis covered a larger period range. In the preliminary analysis, there appear strong peaks around 2.5 , which can however be identified as artifacts originating from the data sampling and the harmonics content in the method (see Sect. 2.4 in You et al. 2000). For the correlation in time (see You et al. 2000), we adopted
$D_{\min }=0.9 \cdot P_{\min }$ and $D_{\max }=t_{\max }-t_{\min }$ for a maximum use of data points. For the maximum correlation in phase $\tau$, we set $\tau=0.125$, allowing a more complicated light curve than the simplest sinusoid of the first order.

Figure 2 shows the multichannel $(U B V R) D(f)$ for data set S1 from Mt. Maidanak. The upper spectrum is derived from the original data and reveals a frequency of $0.216 \mathrm{day}^{-1}$ as the deepest minimum; the corresponding period after refinement with LM and NLM is $4.6261 \pm 0$ d 0070 . The lower spectrum is derived from the residuals of S1 after the NLM fit. Unlike the upper spectrum, the lower one contains only noise and thus shows that there are no significant additional periods. This conclusion is also supported by the fact that the minima in the four $D(f)$ spectra obtained for single channels do not appear at the same frequencies.

Figure 3 shows the multichannel $D(f)$ for the $\mathrm{S} 4$ Mt. Maidanak data set. The top spectrum reveals a period of $5.1578\left(f=0.1939 \mathrm{day}^{-1}\right)$. The middle spectrum is obtained 

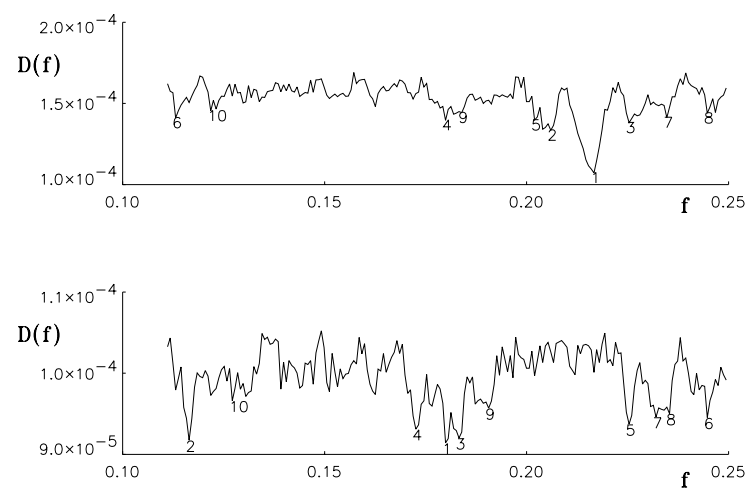

Fig. 2. The multichannel (UBVR) PDM spectra of S1 (Mt. Maidanak). The numbers label the depth ranks of the valleys in the spectra. The unit of frequency $f=1 / P$ is cycle/day. Notice the change of scale in $D(f)$. See explanations in the text.
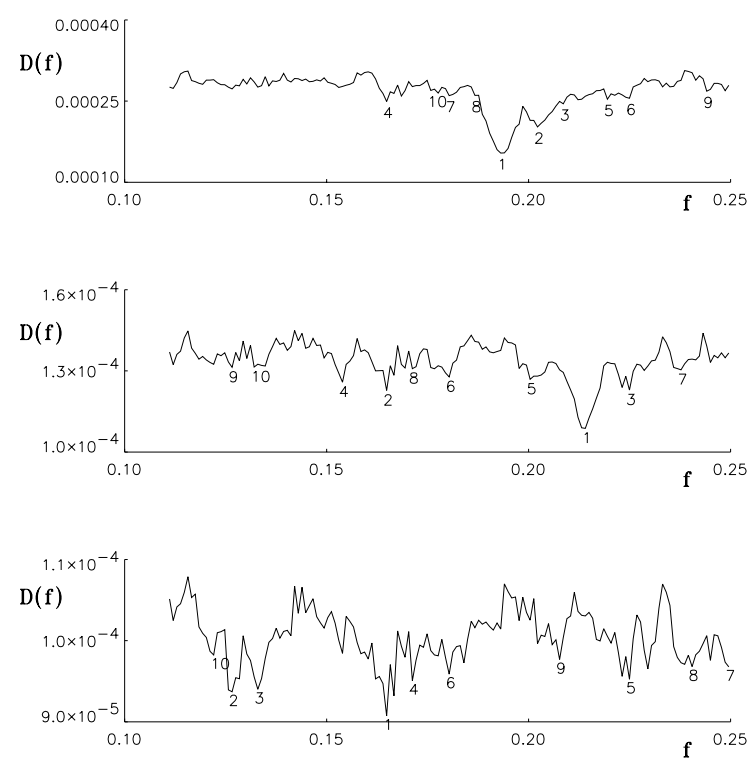

Fig. 3. The multichannel ( $U B V R$ ) PDM spectra of S4 (Mt. Maidanak). Notice the change of scale in $D(f)$. See explanations in the text.

from the residuals after the NLM fit. Here, a second period of $4.7062\left(f=0.2125 \mathrm{day}^{-1}\right)$ is clearly seen. The bottom spectrum is obtained from the residuals after the first and the second periods have been subtracted. Here, we interpret the deepest minimum as due to the noise because of its closeness in depth to other minima and because it is present in only one channel. Thus, there are no significant periods left.

Figure 4 shows the multichannel (by) $D(f)$ spectra for the $\mathrm{s} 2 \mathrm{~b}$ (APT) data set. The top spectrum reveals the first period of 4.6288 $\left(f=0.2160 \mathrm{day}^{-1}\right)$. The second spectrum is obtained from the residuals after the NLM fit. Here, the second (only slightly longer) period of $4.7548\left(f=0.2103\right.$ day $\left.^{-1}\right)$ appears as the deepest minimum in the spectrum. The third spectrum is obtained from the residuals after the first and the second periods have been subtracted. Its deepest minimum is located at the period of $5.0887\left(f=0.1965\right.$ day $\left.^{-1}\right)$. This period is also present in the S2 (Mt. Maidanak) data set which overlaps in time with $\mathrm{s} 2 \mathrm{~b}$ (Fig. 1). The bottom spectrum is obtained from the residuals after subtracting the model containing the three
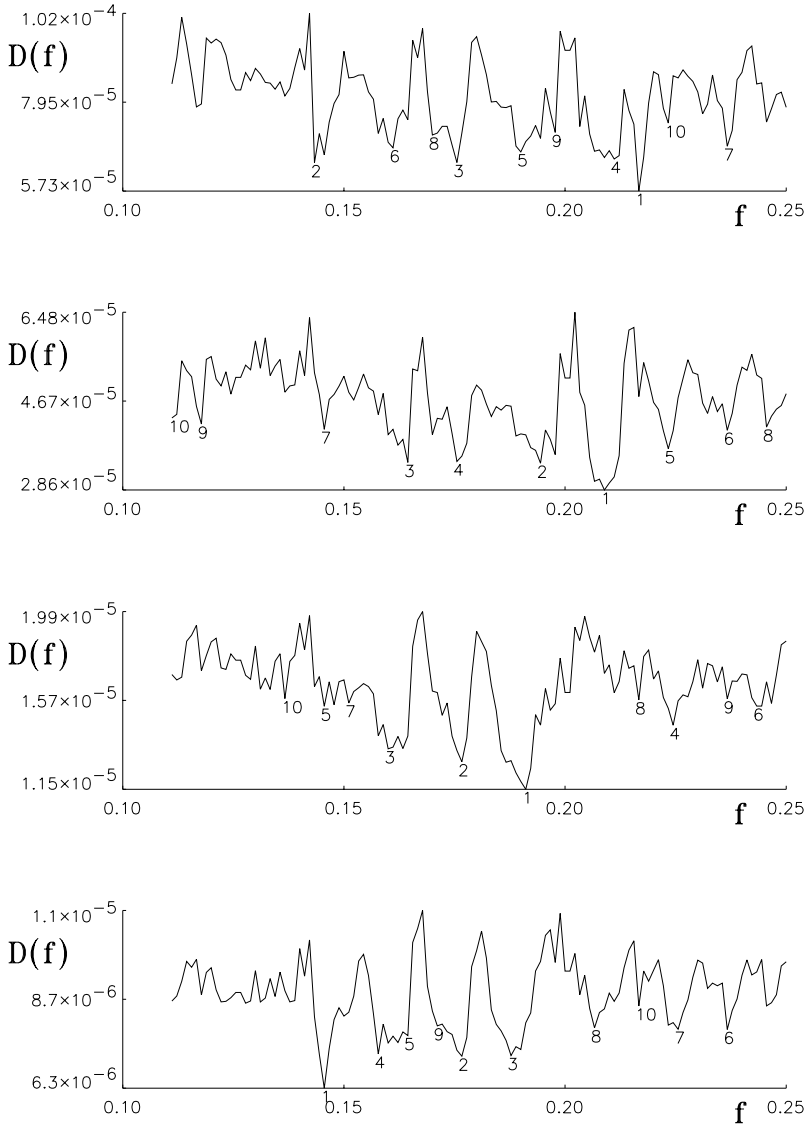

Fig. 4. The multichannel (by) PDM spectra of s2b (APT). Notice the change of scale in $D(f)$. See explanations in the text.

(fixed) periods in the LM fit. The deepest peak around a period of $6.9\left(f=0.15 \mathrm{day}^{-1}\right)$ has been checked carefully. After analyzing the time spacing of the data in the set $\mathrm{s} 2 \mathrm{~b}$ and checking the phase process diagrams (PPDs) folded with this period, this minimum has been identified as arising from the uneven data sampling and the noise fluctuations. Thus, no significant fourth period can be identified. This is the only season where more than two significant periods are present.

The second stage of LM and the last stage of NLM use the same second order sinusoidal model. LM determines a more accurate value of the period than can be obtained by PDM. NLM optimizes the final value of the period using the LM refined value as the start value and also gives error estimates for the period from the curvature of the $\chi^{2}$ hypersurface. The model for fitting the original data with only one period is

$M^{c}\left(t, \beta^{c}(P)\right)=A_{0}^{c}+\sum_{r=1}^{R}\left[A_{r}^{c} \cos (2 \pi r f t)+B_{r}^{c} \sin (2 \pi r f t)\right]$,

where $f=1 / P$ is the trial frequency and $\beta^{c}(P)$ includes all linear parameters $A_{0}^{c}, A_{r}^{c}, B_{r}^{c}$. Here, $A_{0}^{c}$ is the mean magnitude and $A_{r}^{c}, B_{r}^{c}$ are the amplitudes of the cosine and sine functions for channel $c$ and harmonic $r$. We choose the order $R=2$ for the trigonometric polynomial in accordance with $\tau=0.125$ chosen in the PDM search. For the full multichannel model, all $\beta^{c}$ of the individual channels are independent, but the period $P$ is the same in all models $M^{c}$ fitted to the full set of observations. 


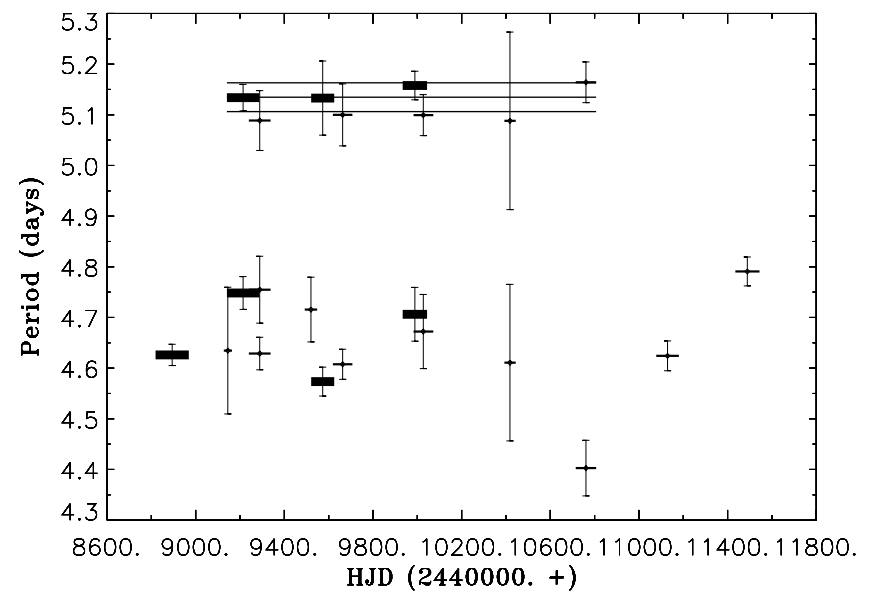

Fig. 5. Periods of HN Peg recovered with MPA from the Mt. Maidanak and APT data. The periods from the multichannel (UBVR) Mt. Maidanak data are plotted as thick horizontal lines whose lengths correspond to the time spans. The periods from the multichannel (by) APT data are plotted as thin horizontal lines. The $3 \sigma$ error bars are also plotted for each period. The upper three long horizontal lines indicate the weighted average period of 5.1348 and its $3 \sigma$ error limits, and show the time span over which the period is detected.

Tables 1 and 2 give the results of MPA for the Mt. Maidanak and the APT data sets. Each final period $P$ and its error estimate $\sigma$ are optimized with NLM. The amplitudes for each period and each channel are obtained from the fits of the multiple period model. Since the model contains two harmonics for each period, the amplitudes given in Tables 1 and 2 for channel $c$ are defined as the magnitude differences between the maximum and the minimum of the model curves.

Figure 5 plots our periods. For the Mt. Maidanak data, S2, $\mathrm{S} 3$ and $\mathrm{S} 4$ contain two periods. The longer period is virtually identical in S2, S3 and S4. The shorter period, which is the only period detectable in S1, appears to be variable from season to season. For the APT data, the longer period is detected in $\mathrm{s} 2 \mathrm{~b}$, $\mathrm{s} 3 \mathrm{~b}, \mathrm{~s} 4 \mathrm{~b}, \mathrm{~s} 5$ and $\mathrm{s} 6$. This longer period does not change with time and its value is about 5.1 . The shorter period appears in all seasons and varies between 4.4 and 4.8 ; the minimum of 4.4 occurs in $\mathrm{s} 7$ and the maximum of 4.8 in s8. Only in the s $2 \mathrm{~b}$ set are three periods detected, where the two shorter ones are close to each other. Comparing the time spans of the Mt. Maidanak and APT data, one can see that the recovered periods from the two data sets generally agree with each other within the error bars.

The APT and the Mt. Maidanak data show that the longer period in the various seasons agree within the $3 \sigma$ error limits, so we can assume that this period is constant for all seasons where it appears. Therefore we compute a final weighted average value of the longer period from its measured values in $\mathrm{S} 2, \mathrm{~S} 3, \mathrm{~S} 4, \mathrm{~s} 2 \mathrm{~b}, \mathrm{~s} 3 \mathrm{~b}, \mathrm{~s} 4 \mathrm{~b}, \mathrm{~s} 5$ and $\mathrm{s} 6$ (Tables 1 and 2) using the errors to compute the weights:

$\bar{P}_{\text {longer }}=5.1348 \pm 0.0095$ days.

Figure 6 shows the phase process diagrams (PPDs) for S1 and the combination of the S3 and S4 (Mt. Maidanak) data sets. For S1, the PPDs of the four channels are folded with the single
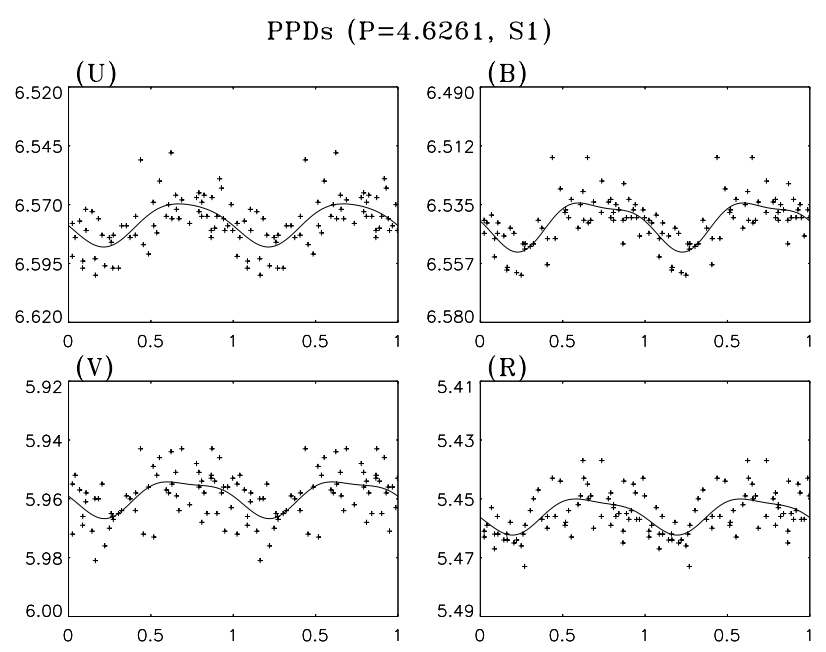

PPDs $(P=5.1348, S 3, S 4)$
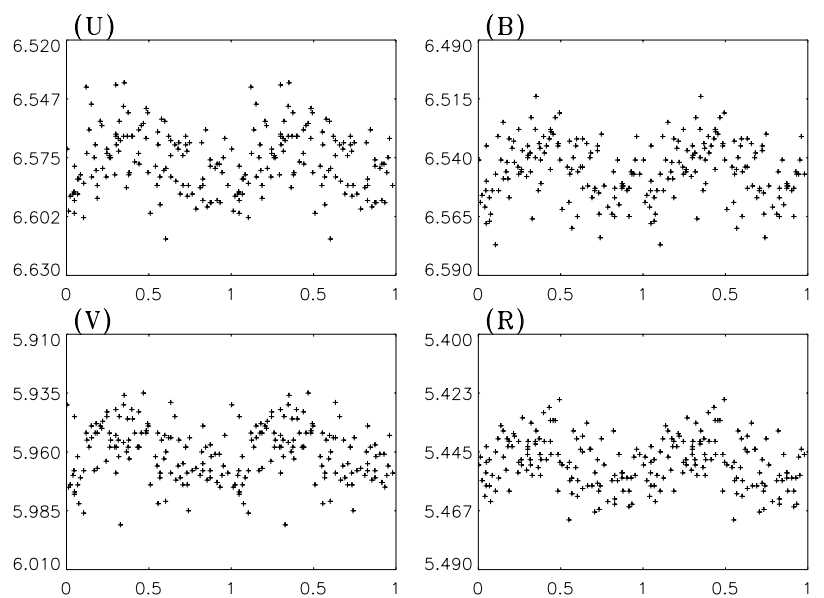

Fig. 6. Phase process diagrams (PPDs) of HN Peg from Mt. Maidanak data (S1, S3 and S4). See text for explanations.

period found in this season, and the final multichannel model obtained from NLM is also plotted. For the combination of S3 and S4 the PPDs are folded with the weighted average period of $5^{\mathrm{d}} .1348$.

Figure 7 shows the PPDs for the APT data. The PPDs of s3a-s6 are created by folding the APT data with the weighted average period of 5.1348 , which is the most significant period in $s 4 b$, s5 and s6. For s8, the PPDs of the two channels are folded with the shorter period, which is the only period detected in that season. The final multichannel model obtained from NLM is also plotted. All phases in the PPDs in Figs. 6 and 7 have been computed with $\phi=0$ at HJD $=2440000$.

Figure 8 shows the PPDs for s5. The PPDs of the longer (main) period are created by folding the original data with the period of 5.0881 detected for this season, while the PPDs of the shorter period are created by folding the residuals after subtracting the longer period. The final multichannel models obtained from NLM are also plotted in the PPDs. This suggests that both detected periods are real.

Figure 9 shows the S4 Mt. Maidanak data set, in which two periods were present, along with the two-period model. 

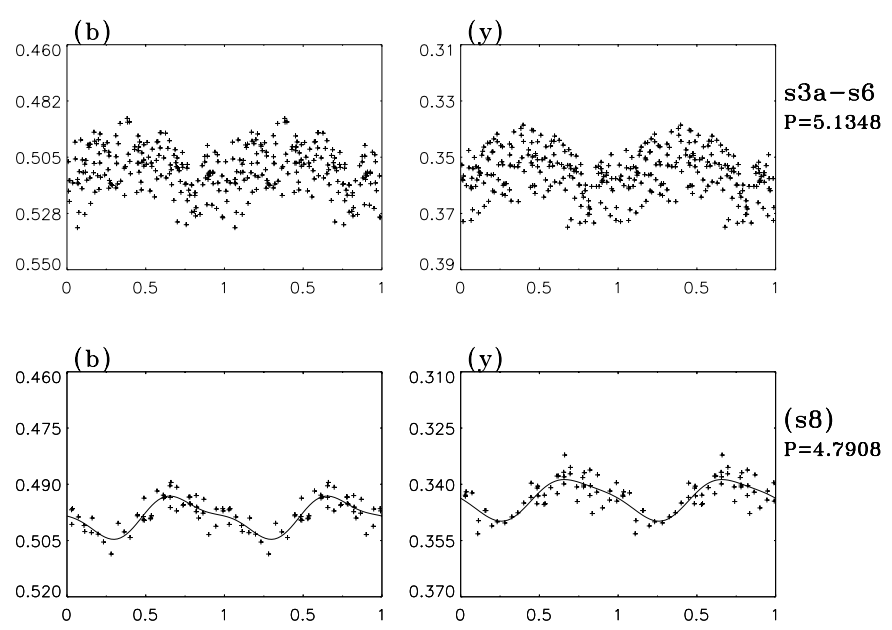

Fig. 7. PPDs of HN Peg from APT data (s3a-s6 and s8). See text for explanations.
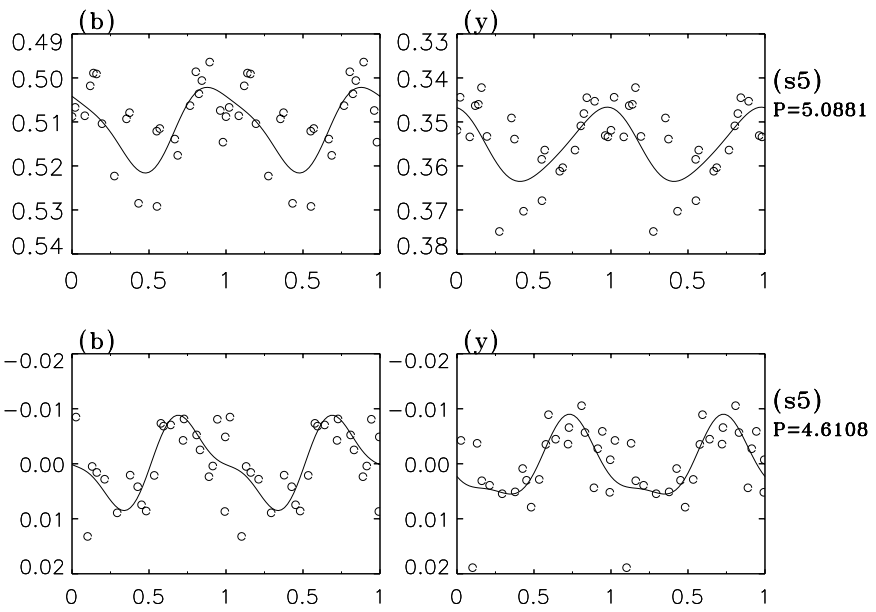

Fig. 8. PPDs of HN Peg from APT data (s5). See text for explanations.

The variation of the amplitudes of the multichannel light curves is well reproduced by the model.

Figure 5 showed that there seems to be a constant value for the longer period during all the time it was present. This longterm constant period allows us to look for systematic behaviour in the active zone responsible for the light variations. For this purpose we determine the phases of the light curve minima resulting from the active zone responsible for the longer period. A two-period model is fitted to the original data, fixing the shorter period to the values given in Tables 1 and 2 and the longer period to the weighted average of 5.1348 . From this model, the phases of the light curve minima resulting from the 5 day variations have been computed and averaged over the 4 and 2 channels, respectively, using the formal errors of the channels as weights. The average phases are given in Table 3 and plotted versus time in Fig. 10.

The accuracy of the phases can be judged from the fact that the phases derived from the independent data sets S3 and s3b as well as $\mathrm{S} 4$ and s4b agree very well. The phase jump between $\mathrm{S} 2$ and s2b arises most likely from the fact that the light variation at the longer period has just become stable during S2 and $\mathrm{s} 2 \mathrm{~b}$, and the two seasons are not overlapping much. Thus, the

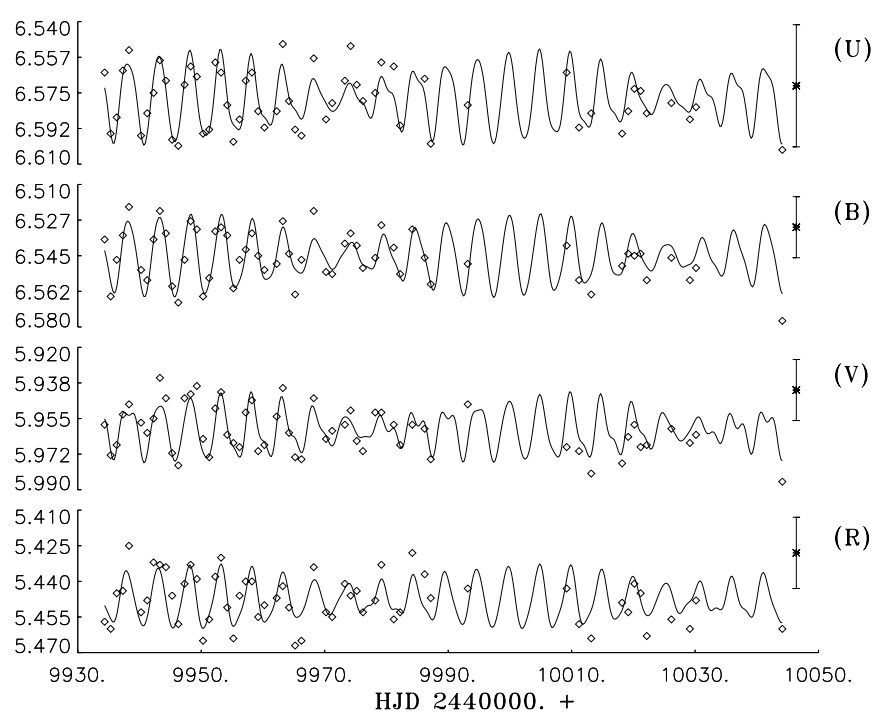

Fig. 9. The observations and modeling curves of S4 (Mt. Maidanak). The full model consists of the two periods (two harmonics for each) recovered with MPA. The asterisks with vertical bars in the upper right of each plot indicate the observational errors given by Shevchenko (1980) in the corresponding channels.

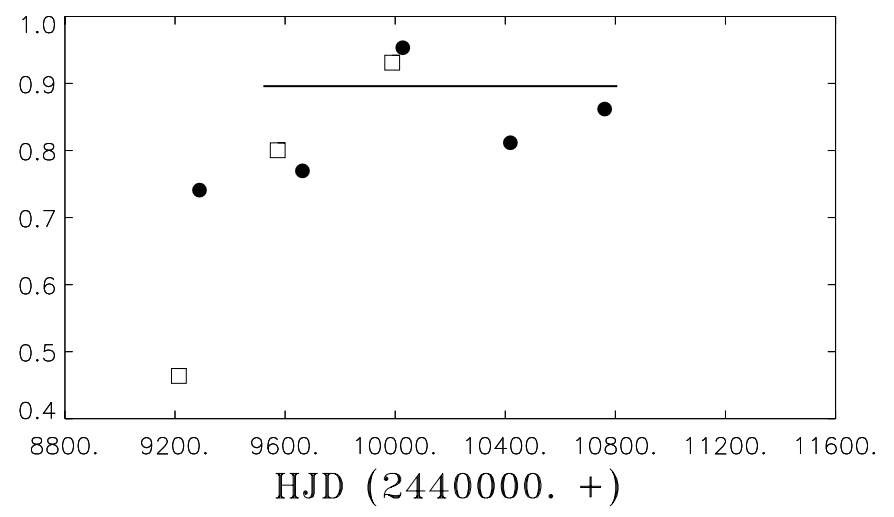

Fig. 10. Phases of the main minima of the light curves with the weighted average period of 5.1348 of the Mt. Maidanak data sets S2, S3, S4 (squares) and the APT data sets s2b, s3b, s4b, s5 and s6 (dots). The horizontal line indicates the weighted average phase of 0.896 during S3, s3b, S4, s4b, s5 and s6.

Table 3. Phases of the main minima of the light curves with the weighted average period of $5^{\mathrm{d}} .1348$.

\begin{tabular}{cccccccc}
\hline \hline S2 & s2b & S3 & s3b & S4 & s4b & s5 & s6 \\
\hline 0.46 & 0.74 & 0.80 & 0.77 & 0.93 & 0.95 & 0.81 & 0.86 \\
\hline
\end{tabular}

deviation of $S 2, S 4$, and s4b from the trend delineated by the other points is probably real. Therefore, there is no simple behaviour of the phase over time, even when the period is virtually constant.

\section{Discussion}

1. Our MPA results obtained separately for the Mt. Maidanak and APT data agree well with each other. We detect two periods in most observing seasons. The longer period near 5.1348 appeared around mid-1993; it is first seen in S2 


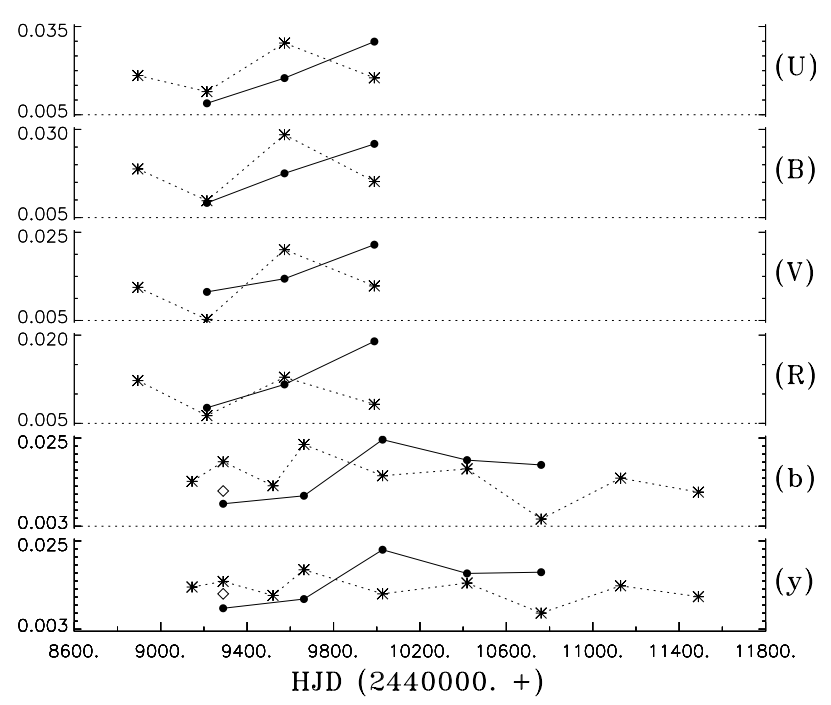

Fig. 11. Amplitudes of the model curves of Mt. Maidanak (UBVR) and APT (by) data. The dotted lines connect asterisks, which are the amplitudes of the shorter periods. The full lines connect the dots, which are the amplitudes of the longer period. The diamonds in the plots for $b$ and $y$ are the amplitudes of the additional period of 4.7548 detected only in s2b. See Tables 1, 2 and Fig. 5.

(Mt. Maidanak in Table 1) and confirmed by s2b (APT in Table 2). This period remained constant from season to season until it disappeared in 1998. A variation with a shorter period was present in all observing seasons with periods in the range $4.4-4.8$. Both the longer and the shorter periods are found also if the entire data set is analyzed together (see point 5 below). Because the two periods are well separated, they are not likely to misleadingly interfere with each other.

2. The brightest $V$ magnitude, the $B-V$ colour index, and the parallax from Hipparcos, combined with the $B-V$ vs. $T_{\text {eff }}$ relationship of Flower (1996), lead to a stellar radius of about $1.03 R_{\odot}$ for HN Peg. Together with $v \sin i=$ $11 \mathrm{~km} \mathrm{~s}^{-1}$ (Kraft 1967), this radius implies an upper limit for the rotation period of $4.7 \pm 0.5$, where we assumed a total error of $10 \%$. Therefore, our shorter periods for HN Peg are consistent with this upper limit only for a high inclination $i$. The longer period is just inside the adopted $1 \sigma$ range, while the exceptional period of 5.7 observed by Donahue et al. (1996) in 1984 is marginally in conflict with the above upper limit.

3. Our MPA results support the presence of two simultaneous periods in at least some of the observing seasons. This may be interpreted as originating from differential rotation in HN Peg (see also point 5) where the longer period originates from a surface structure that has a different rotation rate than the region responsible for the shorter period. The PPDs of the combination of the S3 and S4 Mt. Maidanak data sets (Fig. 6) and the PPDs (s3a-s6) of the APT data sets (Fig. 7) show an identical pattern, from which a maximum and a minimum can be recognized. Here, one should notice that the original data contain not only noise, but even more importantly the variations arising from the other, shorter period, as well as the phase shifts seen in Fig. 10. We still see a clear pattern with a minimum close to the average phase indicated in Fig. 10, despite the long time span of 1315 days (HJD $2449492-H J D 2450807$ ). One possibility is that the new surface structure responsible for the longer period is very long-lived and stays more or less at the same location from its formation until its disappearance (see the discussion in Eaton et al. 1996). It may also indicate that several shorter-lived structures formed during this long timespan more or less at the same location, apart from moderate shifts in longitudes. Baliunas et al. (1998) detected a very significant increase in the activity of the relative $\mathrm{Ca}$ II $\mathrm{H} \& \mathrm{~K}$ emission flux of $\mathrm{HN}$ Peg during about the same time as our S4 and s4b data sets (see their Fig. 7). This may correspond to the activity of the surface structures with the longer period reaching its maximum. At that time, the amplitude is much higher than that of the shorter period and becomes the highest among the seasons.

4. From their Ca II H\&K data, Baliunas et al. (1985) also found two periods: the shorter one at $4.65-4.68$ seems to be more stable than ours and the longer one changes significantly before vanishing between 1981 and 1982. In our data, the longer period is present between 1993 and 1998 and is virtually constant with a stable light curve during its entire lifetime. While in our data the amplitude of the brightness variation with the longer period is steadily increasing until it becomes the dominant variation in 1995 (Fig. 11), Baliunas et al. (1985) found their longer period to be always weaker than the shorter one between 1980 and 1982. It seems that the activity with the shorter period is permanent, while the activity with the longer period is not always present. It would be interesting to have more data coverage on a long time scale to investigate a possible cycle in the appearance and disappearance of the longer period.

5. Both the shorter and longer periods have also been found in the whole Mt. Maidanak and APT data sets. The MPA analysis of the whole data sets was done as follows: First, the mean magnitudes of each season were subtracted, in order to eliminate the long term brightness trends in the data. Next, the procedures for the identification, final optimization, and subtraction of the first period were performed in the same way as described in Sect. 3. The shorter and the longer periods found were 4.6412 \pm 0.0013 and $5.1512 \pm$ 0.0014 from the Mt. Maidanak data, and $4.64573 \pm 0.00052$ and $5.13754 \pm 0.00087$ from the APT data. The value of the longer period agrees with the weighted averages from the individual seasons $(5.1348 \pm 0 \mathrm{~d} .0095)$ within the $3 \sigma$ uncertainties. After subtracting the two main periods from the PDM spectra, some residual power remains close to the shorter period: from this a third period may be obtained as was done for the individual season s2b (Fig. 4 and Table 2). The presence of significant power on a broad frequency range around the shorter period is in agreement with the result of our analysis of individual seasons, giving different shorter periods within a large period range 4.4-4.8.

6. The basic result from our MPA analysis of HN Peg is that we have resolved two distinct photometric periods, which imply two separate latitude zones of activity on the star. Besides interpreting the two distinct periods as arising from active zones rotating differentially with respect to 
each other, Baliunas et al. (1985) also give an alternative interpretation. They propose that the growth and decay of the active regions with subsequent birth of new active regions at stellar longitudes different from the original sites of activity introduces a phase shift, leading period finding algorithms to identify more than one period, none of which may represent the true period. MPA is based on the assumption that Eq. (1) is correct in the subsets of the Mt. Maidanak and APT data (see their time spans in Tables 1 and 2). This assumption is based on constant periods and no phase shifts/jumps for each period in the light curves. Our MPA results are correct only in so far as this assumption is correct. For testing the second alternative interpretation we have also done simulations based on light curves with a phase shift. The noise-free light curves consisted of a sinusoid sampled in the same way as one of our observing seasons. Half way through the season, a phase shift of 0.5 was introduced. Our MPA analysis for these light curves show that the phase shift does introduce additional periods in the PDM and LM spectra because of the mismatch of Eq. (1) to the simulated light curves. The existence of a phase shift can easily be identified by constructing the PPDs for the 2 halves of the season separately. We have also checked for these kinds of phase shifts in PPDs for all subsets of both Mt. Maidanak and APT data folded with the shorter period, the longer period, and the mean of the two periods, respectively. Our results show that phase shifts could not be identified in HN Peg so that the two periods found cannot be explained by this alternative interpretation.

7. This is the first application of MPA (You et al. 2000) to real observations. Although the method was originally developed for the case of one period only, the first stage of PDM search is able to identify multiple periods by analyzing the residuals after subtracting the previously known periods. The additional stages of LM and NLM are able to constrain the periods to a satisfactory degree through a onedimensional refinement, which optimizes only one period in the original data and interprets the effects from other periods as random noise. We have done simulations based on the HN Peg data and shown that, statistically, the periods from the minima of the one-dimensional $\chi^{2}$ (as used in this paper) are indistinguishable from those from the minimum of the two-dimensional $\chi^{2}$. For treating multiple period cases, complete modeling with nonlinear multi-period
LSQ fit will achieve more precise final solutions than the present single period fitting, which may give somewhat biased period values because of the scarcity of the data. The full multiple-period NLM iteration is under construction and testing, and will be reported on in future studies. At present the evaluation of the significance of minima in the PDM spectra and thus the presence of the periods relies on a visual comparison of the multichannel spectrum and the single channel spectra. This process is somewhat subjective. To obtain a more quantitative measure of the significance of PDM minima, the use of the bootstrap method for statistical error estimates is planned.

Acknowledgements. The authors thank Dr. V. S. Shevchenko for providing the Mt. Maidanak photometric data, Dr. Andrei Berdyugin for helping with the data and checking the text and Dr. Jaan Pelt for many valuable discussions. We also thank G. Henry for providing the APT data.

\section{References}

Baliunas, S. L., Donahue, R. A., Soon, W., \& Henry, G. W. 1998, in Cool Stars, Stellar Systems and the Sun, Tenth Cambridge Workshop, ed. R. A. Donahue, \& J. A. Bookbinder, ASP Conf. Ser., 154, 153

Baliunas, S. L., Horne, J. H., Porter, A., et al. 1985, ApJ, 294, 310

Donahue, R. A., Saar, S. H., \& Baliunas, S. L. 1996, ApJ, 466, 384

Eaton, J. A., Henry, G. W., \& Fekel, F. C. 1996, ApJ, 462, 888

Flower, P. J. 1996, AJ, 469, 355

Frasca, A., Freire Ferrero, R., Marilli, E., \& Catalano, S. 2000, A\&A, 364,179

Gaidos, E. J. 1998, PASP, 110, 1259

Gaidos, E. J., Henry, G. W., \& Henry, S. M. 2000, AJ, 120, 1006

Henry, G. W. 1999, PASP, 111, 845

Henry, G. W., Eaton, J. A., Hamer, J., \& Hall, D. S. 1995, ApJS, 97, 513

Jetsu, L., Hackman, T., Hall, D. S., et al. 2000, A\&A, 362, 223

Kraft, R. P. 1967, ApJ, 150, 551

Messina, S., \& Guinan, E. F. 2002, A\&A, 393, 225

Messina, S., \& Guinan, E. F. 2003, A\&A, 409, 1017

Shevchenko, V. S. 1980, Astr. Zh., 57, 1162 (SvA, 24, 670)

Vaughan, A. H., Baliunas, S. L., Middelkoop, F., et al. 1981, ApJ, 250, 276

Wilson, O. C. 1978, ApJ, 226, 379

You, J., Pelt, J., \& Tuominen, I. 2000, A\&AS, 146, 397 